\title{
Crianças e Adolescentes Institucionalizados: Desempenho Escolar, Satisfação de Vida e Rede de Apoio Social ${ }^{1}$
}

\author{
Aline Cardoso Siqueira \\ Centro Universitário Franciscano \\ Débora Dalbosco Dell'Aglio ${ }^{2}$ \\ Universidade Federal do Rio Grande do Sul
}

\begin{abstract}
RESUMO - Este estudo objetivou investigar as características de jovens institucionalizados e suas famílias. Participaram 155 crianças e adolescentes, de 7 a 16 anos, de instituições da Região Metropolitana de Porto Alegre/RS. A coleta de dados foi realizada por meio de entrevista estruturada, Teste de Desempenho Escolar, Escala de Satisfação de Vida e Mapa dos Cinco Campos. Foi observada a presença precoce de experimentação de drogas, baixo desempenho escolar e alto índice de repetência entre os jovens. As famílias apresentaram baixa escolaridade, trabalhos informais e desemprego. Contatos positivos e alta satisfação de vida na instituição podem indicar que o acolhimento institucional se constitui em fonte de apoio e satisfação. Programas de intervenção para o desenvolvimento desses jovens e fortalecimento das famílias são discutidos.
\end{abstract}

Palavras-chave: institucionalização; desempenho escolar; satisfação de vida; rede de apoio social.

\section{Institutionalized Children and Adolescents: Academic Achievement, Life Satisfaction and Social Support Network}

\begin{abstract}
This study aimed to investigate the characteristics of institutionalized youth and their families. The participants were 155 institutionalized children and adolescents, aged from 7 to 16 years old, from shelters in the metropolitan region of Porto Alegre/RS. Data collection was accomplished through structured interviews, the Academic Achievement Test, the Life Satisfaction Scale and the Five Field Map. Early experimentation of drugs, poor school performances and high levels of school failure were observed among the adolescents. Families presented low educational levels, informal jobs and unemployment. Positive contacts and high life satisfaction in the institution may indicate that the institutional acceptance constitutes a source of support and satisfaction. Intervention programs for the development of those youth and for empowerment of their families are discussed.
\end{abstract}

Keywords: institutionalization; academic achievement; life satisfaction; social support network.

A problemática da institucionalização na infância e na adolescência constitui-se em um tema de grande importância social. Sua relevância deve-se não somente ao grande número de jovens em situação de institucionalização, cerca de 20.000, segundo Levantamento Nacional de Abrigos Brasileiros para Crianças e Adolescentes (Silva, 2004), mas também à preocupação relacionada à qualidade de atendimento oferecido nos atuais acolhimentos institucionais e à necessidade de oportunizar o desenvolvimento humano e a construção da cidadania. Após a implementação do Estatuto da Criança e do Adolescente - ECA (Brasil, 1990), mudanças têm sido constatadas nos acolhimentos institucionais, tanto no que concerne à organização quanto à estrutura física, buscando uma melhor qualidade no seu atendimento (Guará, 2006;

1 Esse artigo é parte da Tese de Doutorado intitulada "Crianças, adolescentes e transições ecológicas: Instituições de abrigo e família como contextos de desenvolvimento", de autoria da primeira autora, sob orientação da segunda. Apoio do Conselho Nacional de Desenvolvimento Científico e Tecnológico $(\mathrm{CNPq})$ e da Fundação de Amparo à Pesquisa do Rio Grande do Sul (FAPERGS).

2 Endereço para correspondência: Instituto de Psicologia, UFRGS. Ramiro Barcelos, 2600. Porto Alegre, RS. CEP 90035-003. Fone: (51) 3308-5253; Fax: (51) 3308-5473. E-mail: dalbosco@cpovo.net.
Siqueira \& Dell'Aglio, 2006). Considerando esse panorama, tornam-se necessárias pesquisas que busquem investigar as características e o desenvolvimento das crianças e adolescentes que vivem atualmente nos acolhimentos institucionais brasileiros.

As legislações que têm norteado o funcionamento das instituições que atendem crianças e adolescentes afastados do convívio familiar são o ECA (Brasil, 1990) e, mais recentemente, a Lei ${ }^{\circ} 12.010$, conhecida como Lei Nacional da Adoção (Brasil, 2009). De acordo com o ECA, o abrigamento é uma medida de proteção, de caráter provisório e excepcional, utilizada sempre que os direitos das crianças e adolescentes são ameaçados ou violados. Envolve o afastamento da criança ou adolescente da convivência familiar e a passagem da guarda provisória desses para o dirigente da instituição. A nova Lei Nacional da Adoção aperfeiçoou as determinações do ECA, preconizando que, em regra, o tempo de afastamento da família não pode ultrapassar dois anos. Além disso, o termo "abrigamento" foi substituído por "acolhimento institucional" (Brasil, 2009), entre outras determinações.

Ainda não se pode avaliar o impacto da nova Lei Nacional da Adoção, contudo, pode-se constatar que o ECA 
promoveu reformulações nas instituições, atuando tanto na redução das instalações físicas das instituições, que nas décadas anteriores atendiam coletivamente cerca de 30 a 50 crianças e adolescentes (Rizzini \& Rizzini, 2004), como na construção e implementação de um programa socioeducativo. Esse programa deveria buscar o desenvolvimento pleno dos abrigados, vendo-os como seres humanos em desenvolvimento, com potencialidades e limitações, dissociando a institucionalização da função exclusivamente assistencialista e da ideia de depósito de jovens "problemáticos" (Guará, 2006).

Segundo as diretrizes do ECA, os acolhimentos institucionais devem assumir caráter residencial, oferecendo atendimento personalizado, em pequenas unidades e grupos reduzidos. Também deveriam disponibilizar um ambiente acolhedor, satisfatório e propício ao desenvolvimento da autonomia e da identidade (Arpini, 2003). Assim, a instituição pode ser caracterizada como um contexto de desenvolvimento, proporcionando o provimento material e um ambiente seguro e afetivo (Siqueira, Betts \& Dell'Aglio, 2006; Yunes, Miranda \& Cuello, 2004).

Inúmeras pesquisas têm sido realizadas com o intuito de conhecer como está sendo o desenvolvimento e qual é a percepção das crianças e adolescentes institucionalizados quanto, por exemplo, a si mesmos, ao acolhimento institucional, sua visão de família, eventos de vida, envolvimento em brincadeiras lúdicas e percepção de rede de apoio (Arpini, 2003; De Antoni \& Koller, 2000; Dell'Aglio, 2000; Dell'Aglio \& Hutz, 2004; Martins \& Szymanski, 2004; Oliveira, 2006; Pasian \& Jacquemin, 1999; Silva, 2004; Siqueira \& cols., 2006). O desenvolvimento cognitivo constitui-se em um relevante campo a ser pesquisado em crianças e adolescentes institucionalizados. O desempenho escolar e o nível intelectual são considerados fatores individuais que podem moderar os efeitos negativos do estresse, operando como fator de proteção (Garmezy, Masten \& Tellegen, 1984) e atuando como promovedor de inclusão social (Guará, 2006). Estudos apontam que crianças que tiveram uma vida difícil ou problemas nas relações parentais podem apresentar dificuldades de adaptação e de aproveitamento na escola (Ferreira \& Marturano, 2002).

A satisfação de vida é outro aspecto importante a ser investigado entre as crianças e adolescentes afastados da família. É um componente do bem-estar subjetivo e está relacionada à avaliação cognitiva global, aos julgamentos que um indivíduo faz sobre sua própria vida, envolvendo aspectos racionais e intelectuais (Ryff \& Keyes, 1995). Além de uma avaliação global, o indivíduo também pode emitir julgamentos de domínios específicos de sua vida, como trabalho, escola, amizades, amor, entre outros (Lucas, Diener \& Suh, 1996). Entre os fatores associados à satisfação de vida, encontra-se a rede de apoio social.

Rede de apoio social é definida como conjunto de sistemas e de pessoas significativas que compõem os elos de relacionamento recebidos e percebidos do indivíduo (Brito \& Koller, 1999). A rede de apoio social está associada à saúde e ao bem-estar dos indivíduos (Samuelsson, Thernlund \& Ringström, 1996; Sluzki, 1997), sendo um fator fundamental para o processo de adaptação a situações de estresse e de suscetibilidade a distúrbios físicos e emocionais (Masten \& Garmezy, 1985), atuando como fator de proteção. Para as crianças e adolescentes que vivem em acolhimentos institucionais, as pessoas com as quais eles convivem passam a integrar as suas redes de apoio social e afetivo, fazendo da instituição o seu ambiente principal (Siqueira \& cols., 2006; Yunes \& cols., 2004). É no acolhimento institucional que realizam um grande número de atividades, desempenham papéis e funções e interagem, abrindo espaço para o desenvolvimento de relações recíprocas, de equilíbrio de poder e de afeto estável. Assim, embora a institucionalização geralmente esteja relacionada a sofrimento, os acolhimentos institucionais podem proporcionar um espaço seguro e protetivo, possibilitando acolhimento e relações satisfatórias (Siqueira \& cols., 2006).

Apesar das mudanças no panorama dos acolhimentos institucionais, existe ainda uma carência de estudos no Brasil que forneçam dados específicos sobre as características dos jovens abrigados e suas famílias nos anos atuais. Não foram encontrados estudos, por exemplo, sobre satisfação de vida em jovens abrigados na realidade brasileira. Assim, a partir da perspectiva epistemológica da Teoria Bioecológica do Desenvolvimento Humano (Bronfenbrenner, 2004; Bronfenbrenner \& Morris, 1998), procurou-se investigar duas das quatro dimensões do modelo bioecológico (PPCT - Pessoa, Processo, Contexto e Tempo), a pessoa e o contexto. A dimensão pessoa refere-se às características individuais, físicas e psicológicas do indivíduo em desenvolvimento, aspectos cruciais para a sua relação com o mundo social. Já a dimensão contexto inclui tanto os contextos de interação face a face do indivíduo quanto aqueles mais amplos, como sociedade, valores e cultura. Essa dimensão é compreendida a partir da interação dos quatro níveis ambientais: microssistema, mesossistema, exossistema e macrossistema, e constitui seu ambiente ecológico (Bronfenbrenner, 1979/1996).

Dessa forma, o objetivo do presente estudo foi investigar as características das crianças e adolescentes institucionalizados quanto a: (1) variáveis individuais, como experimentação de drogas, satisfação de vida e desempenho escolar (pessoa); (2) variáveis familiares, como características das famílias; e (3) variáveis sociais, como a rede de apoio social (contexto: microssistema da família, acolhimento institucional, escola, entre outros).

\section{Método}

\section{Participantes}

Participaram deste estudo transversal 155 crianças e adolescentes institucionalizados, de ambos os sexos, de 7 a 16 anos $(M=11,72 ; D P=1,97)$, sendo que $82 \%$ estavam em acolhimentos institucionais governamentais e $18 \%$ estavam em acolhimentos institucionais não-governamentais da Região Metropolitana de Porto Alegre/RS. Eram jovens afastados do convívio familiar, por medida de proteção judicial, em função de maus-tratos, negligência, abandono, violência física, sexual e psicológica. A média de tempo de institucionalização desses jovens era de 35,3 meses $(D P=35,14)$, variando entre 1 mês e 149 meses. O critério de inclusão utilizado foi ter idade entre 7 e 16 anos e o critério de exclusão foi possuir 
alguma deficiência intelectual que pudesse interferir no entendimento dos instrumentos. Essa informação foi obtida com os técnicos dos acolhimentos institucionais. Embora a amostra não tenha sido aleatória, ela representou $82 \%$ do total de jovens abrigados, dessa faixa etária, na Região Metropolitana de Porto Alegre.

\section{Instrumentos}

Foram utilizados os seguintes instrumentos:

1) Entrevista estruturada. Objetivou coletar dados biosociodemográficos dos participantes e suas famílias, como sexo, idade, escolaridade, contato com a família, experimentação de drogas, ocupação dos pais, entre outros;

2) Teste de Desempenho Escolar - TDE (Stein, 1994). É um instrumento psicométrico que busca oferecer uma avaliação das capacidades essenciais para o desempenho escolar por meio dos subtestes de escrita, aritmética e leitura (Alpha de Cronbach total=0,98);

3) Escala Multidimensional de Satisfação de Vida - EMSV (Giacomoni, 2002; Giacomoni \& Hutz, 2008). É uma medida multidimensional de 50 itens, cujo objetivo é avaliar a satisfação de vida, apresentando consistência interna adequada (Alpha de Cronbach=0,93) e correlações apropriadas com outras medidas (Giacomoni \& Hutz, 2008). A escala fornece um escore que varia de 1 a 5 (Escala Likert de cinco pontos). Assim, quanto mais próximo de 5 for a média, maior será a satisfação de vida;

4) Mapa dos Cinco Campos (Samuelsson \& cols., 1996; adaptado por Hoppe, 1998). É um instrumento que avalia estrutura e função da rede de apoio social e afetivo, a partir dos cinco campos: Família, Escola, Amigos, Parentes e Contatos Formais. Baseado no estudo de Siqueira e cols. (2006), o Campo Abrigo foi acrescentado, considerando a importância desse contexto para os participantes. Os campos Amigos e Parentes foram unidos, preservando, assim, os cinco campos (Figura 1). O Mapa dos Cinco Campos é um instrumento lúdico constituído por um pano de feltro e por figuras que podem ser fixadas com velcro, que representam adultos, adolescentes e crianças. $\mathrm{O}$ círculo central corresponde ao participante e cada círculo adjacente mede a qualidade do vínculo, sendo que quanto mais próximo do círculo central for a pessoa colocada, mais satisfação e qualidade há nesse contato. $\mathrm{O}$ último círculo, na periferia do Mapa, corresponde às relações insatisfatórias. Os dados obtidos são anotados em uma folha de registro, conjuntamente com informações sobre satisfação/insatisfação e a existência de conflitos e rompimentos nas relações (Siqueira, Tubino, Schwarz \& Dell'Aglio, 2009). Neste estudo foram utilizadas as seguintes variáveis dependentes: média dos contatos total e nos campos, conflitos, rompimentos e fator de proximidade. $\mathrm{O}$ fator de proximidade é uma variável que representa o grau de vinculação dos participantes com o número de pessoas citadas nos campos, sendo medido por meio da localização dessas pessoas em relação ao círculo central, no qual está o participante. Esse fator varia de 0 a 8 , sendo que escores entre 0 e 2,6 são considerados de pequena força; entre 2,7 e 5,3, média força; e entre 5,4 e 8, grande força de proximidade. Esse escore permite diferenciar qualidade de quantidade, à medida que um participante que mencione 10 contatos em um campo, todos no primeiro nível, terá um maior fator de proximidade nesse campo se comparado a outro participante que mencione os mesmos 10 contatos, entretanto distribuídos nos cinco níveis. A partir do cálculo desse fator, é possível compreender a função da rede, ou seja, a qualidade das relações estabelecidas.

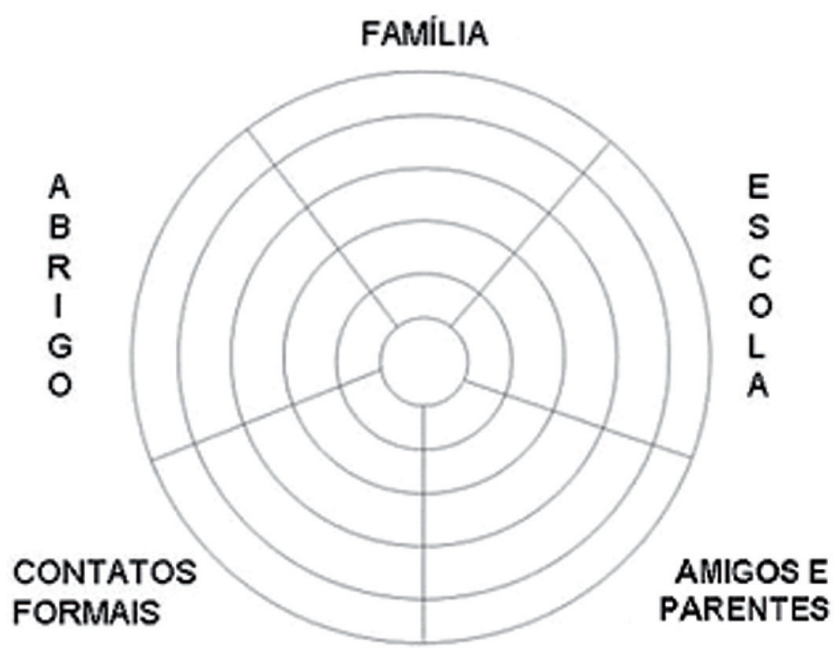

Figura1. Mapa dos Cinco Campos (adaptado por Siqueira, Betts \& Dell'Aglio, 2006).

\section{Procedimento}

Este estudo foi aprovado pelo Comitê de Ética da Universidade Federal do Rio Grande do Sul (processo $\left.\mathrm{n}^{\circ} 2006533\right)$ e as diretorias técnicas dos acolhimentos institucionais autorizaram a sua realização por meio da assinatura do "Termo de Concordância da Instituição". Foram realizados contatos com 10 instituições de acolhimento, governamentais e não-governamentais, sendo que nove permitiram o acesso aos jovens institucionalizados. Das nove instituições, todos os jovens que cumpriam os critérios de inclusão foram convidados a participar do estudo. A coleta de dados foi realizada por uma equipe de pesquisadoras composta por alunas da graduação e pós-graduação do Instituto de Psicologia/UFRGS. Foram realizadas reuniões semanais com a equipe, que recebeu treinamento para o uso dos instrumentos e para sua aplicação. Os participantes responderam aos instrumentos de forma individual em seus horários livres, entre os meses de abril e julho de 2006, em salas cedidas pelos próprios acolhimentos institucionais. 


\section{Análise de dados}

Os dados foram analisados por meio do SPSS for Windows 13.0. Análises descritivas e testes Qui-quadrado foram utilizados para identificar as características biosociodemográficas dos participantes e compará-las por sexo e faixa etária. Correlações de Pearson foram realizadas para verificar relações entre tempo de institucionalização, idade e médias dos instrumentos. Testes $t$ de Student foram utilizados para verificar diferenças entre as médias nos instrumentos por sexo e faixa etária. A magnitude das diferenças entre as médias dos instrumentos nos grupos, para os resultados significativos, foi avaliada usando o effect size de Cohen's $d$. A magnitude foi classificada como pequena, média e grande, segundo Cohen (1998).

\section{Resultados}

A partir da análise da entrevista, foram levantadas as características pessoais dos participantes. A média de irmãos foi de 4,31 $(D P=2,45)$, variando de um a 18 irmãos, sendo que cerca de $60 \%$ dos participantes possuíam irmãos no mesmo local em que estavam abrigados. Das crianças e adolescentes abrigados que mantinham contato com a família $(61,3 \%$ da amostra), seja mãe, pai, tios, avós ou irmãos, 18,7\% mantinham contato esporádico; $12,1 \%$, mensal; $22 \%$, quinzenal; e 47,3\%, semanal. Entre aqueles que não possuíam contato, 93,2\% conheciam os membros da família. Segundo 73,6\% dos jovens, seus pais não viviam mais juntos, contudo não conseguiram informar a atual configuração das suas famílias.

Quanto à escolaridade dos pais, encontrou-se que 44\% dos pais e 53,8\% das mães possuíam o Ensino Fundamental incompleto. Quanto à atividade laboral das figuras parentais, $27,7 \%$ dos pais trabalhavam fazendo biscate e $23,1 \%$ não trabalhavam; 17,6\% das mães trabalhavam em atividades de limpeza e 55,3\% não trabalhavam fora. Testes Qui-quadrado realizados entre as variáveis "contato com a família" e sexo e faixa etária não revelaram relação significativa.

Cerca de 55,5\% dos participantes já experimentaram bebida alcoólica, com idade de início variando entre 5 e 14 anos $(M=9,88 ; D P=2,41) ; 42,6 \%$ já experimentaram cigarro, com idade de início entre 6 e 14 anos $(M=10,37 ; D P=1,84)$; e 14,8\% já experimentaram drogas ilícitas, entre elas loló, cola de sapateiro, maconha, cocaína e crack, com idade de início variando entre 6 e 14 anos $(M=10,48 ; D P=2,15)$. A Tabela 1 apresenta os dados relacionados a sexo e faixa etária. Foi observada relação significativa entre faixa etária e experimentação de álcool ( $\left.\mathrm{c}^{2}=16,16 ; g l=1 ; p<0,01\right)$ e cigarro $\left(c^{2}=19,75 ; g l=1 ; p<0,01\right)$, com um maior uso de ambos entre os adolescentes. Não foram observadas diferenças estatisticamente significativas entre experimentação de álcool, cigarro e drogas ilícitas por sexo.

No que diz respeito à escolaridade, 94,8\% dos participantes estavam matriculados na escola, a maioria estava na $2^{\mathrm{a}} \mathrm{e}$ $3^{\mathrm{a}}$ séries $\left(23,2 \%\right.$ cada), sendo que $90,9 \%$ cursavam até a $5^{\mathrm{a}}$ série do Ensino Fundamental. Quanto à repetência escolar, $72,7 \%$ dos participantes já repetiram de uma a cinco vezes o ano letivo, e desses, 52,7\% eram meninos. No entanto, $64,5 \%$ dos participantes nunca abandonaram a escola e $92,3 \%$ nunca foram expulsos. A Tabela 2 apresenta os resultados do desempenho escolar dos participantes por sexo e faixa etária. Observa-se que as médias foram maiores para as meninas e para os adolescentes em todos subtestes e no total do TDE. As análises indicaram somente diferença significativa entre as médias dos subtestes aritmética $(t=4,31 ; g l=104 ; p<0,01)$, escrita $(t=3,58 ; g l=104 ; p<0,01)$ e leitura $(t=2,71 ; g l=72,01$; $p<0,01)$ por faixa etária, sendo a média dos adolescentes maior do que a média das crianças. $\mathrm{Na}$ análise do tamanho do efeito dessas diferenças, foram encontradas magnitudes grandes no subteste aritmética $(d=0,71)$ e magnitudes médias para os subtestes de leitura $(d=0,47)$ e escrita $(d=0.66)$. Não foi encontrada correlação significativa entre essas variáveis e tempo de institucionalização.

Quanto à satisfação de vida (Tabela 3), não foi observada diferença significativa nem por sexo e nem por idade. Contudo, foi observada uma correlação negativa entre a média total da escala e idade, sendo que quanto maior a idade, menor a média total da EMSV $(r=-0,19 ; p<0,05)$.

A análise estatística do Mapa dos Cinco Campos (adaptado por Siqueira \& cols., 2006) indicou um total de 4420 contatos, sendo 4069 contatos satisfatórios, 351 contatos insatisfatórios, 1054 conflitos e 117 rompimentos. A Tabela 3 apresenta as médias e desvios-padrão dos contatos nos cinco campos do instrumento por sexo e faixa etária. A análise por sexo mostrou que as meninas apontaram maior média de contatos $(t=2 ; g l=124,28 ; p<0,05)$ e de rompimentos $(t=2,65 ; g l=112,46 ; p<0,01)$ no Mapa do que os meninos, com pequena e média magnitudes $(d=0,33$ e $d=0,44$; respectivamente). Quanto à faixa etária, as médias de conflitos $(t=3,71 ; g l=122,35 ; p<0,01)$ e rompimentos $(t=2,01 ; g l=138$; $p<0,05)$ entre os adolescentes foram maiores do que entre as crianças, com média e pequena magnitudes $(d=0,59$ e $d=0,33$, respectivamente).

Testes de Correlações de Pearson foram realizados entre as variáveis total de contatos, contatos satisfatórios, insa-

Tabela 1. Percentual de experimentação de álcool, cigarro e drogas ilícitas por sexo e faixa etária.

\begin{tabular}{lccc}
\hline & Sexo & & Faixa Etária \\
\cline { 2 - 4 } & Meninos $(\mathrm{n}=79)$ & Meninas $(\mathrm{n}=76)$ & Crianças (n=76) \\
\cline { 2 - 4 } Álcool & 57,7 & 53,9 & 38,7 \\
Cigarro & 41 & 44,7 & 24 \\
Drogas Ilícitas & 15,4 & 14,5 & 10,7 \\
\hline
\end{tabular}

Nota: $* p<0,01$ 
Tabela 2. Médias e desvios-padrão dos subtestes do TDE por sexo e faixa etária.

\begin{tabular}{|c|c|c|c|c|c|c|c|c|c|c|}
\hline & \multicolumn{2}{|c|}{ Meninos } & \multicolumn{2}{|c|}{ Meninas } & \multicolumn{2}{|c|}{ Crianças } & \multicolumn{2}{|c|}{ Adolescentes } & \multicolumn{2}{|c|}{ Total } \\
\hline & M & $\mathrm{DP}$ & M & $\mathrm{DP}$ & M & DP & M & DP & $\mathrm{M}$ & DP \\
\hline Aritmética & 11,8 & 6,3 & 12 & 5,6 & 10,6 & 5,2 & $14,7^{*}$ & 4,4 & 11,8 & 5,6 \\
\hline Escrita & 16,8 & 8,8 & 18 & 8,5 & 16 & 7,8 & $21,9^{*}$ & 8,6 & 17,7 & 8,8 \\
\hline Leitura & 51,1 & 20,8 & 53,4 & 18,2 & 50,5 & 19,9 & $59,5^{*}$ & 12,6 & 52,6 & 19,1 \\
\hline Total & 78,8 & 34,5 & 82,8 & 30,8 & 77,1 & 29,1 & 96 & 23 & 81,6 & 31,9 \\
\hline
\end{tabular}

Nota: ${ }^{*} \mathrm{p}<0,01$

tisfatórios, conflitos e rompimentos com idade e tempo de institucionalização. Verificou-se correlação significativa entre idade e média de conflitos, sendo que quanto maior a idade, maior a média de conflitos $(r=0,21 ; p<0,05)$; e entre total de contatos e tempo de institucionalização, sendo que quanto maior o tempo de institucionalização, menor a média de contatos no Mapa $(r=-0,17 ; p<0,05)$. Entre as médias de contatos em cada campo e tempo de institucionalização foi observada correlação significativa somente no Campo Abrigo, sendo que quanto maior o tempo de institucionalização, menor foi a média de contatos no Campo Abrigo $(r=-0,25 ; p<0,01)$.

Para avaliar a qualidade dos elos estabelecidos entre o participante e os contatos mencionados na rede, realizou-se uma análise do fator de proximidade na rede e nos diferentes campos. $\mathrm{O}$ fator de proximidade indica o grau de qualidade dessas relações estabelecidas na rede de apoio. A Tabela 4 apresenta as médias do fator de proximidade nos campos por sexo e faixa etária.

A maioria dos campos apresentou grande força de proximidade (escores entre 5,4 e 8), exceto o Campo Escola para as meninas, Campo Amigos/Parentes para as crianças e os campos Escola e Contatos Formais para os adolescentes (média força). Não foram encontradas diferenças significa- tivas nos campos por sexo e faixa etária. Correlações entre as médias do fator de proximidade nos diferentes campos e tempo de institucionalização foram realizadas, sendo que a única correlação significativa foi com o fator de proximidade do Campo Abrigo. Assim, quanto maior o tempo de institucionalização, menor foi a média do fator de proximidade no Campo Abrigo $(r=-0,19 ; p<0,05)$.

\section{Discussão}

Os resultados deste estudo possibilitaram conhecer algumas características de crianças e adolescentes institucionalizados do sul do Brasil e de suas famílias. No que tange ao microssistema familiar, verificou-se que as famílias dessas crianças e adolescentes são famílias numerosas, com uma média de quatro irmãos, sendo que a maioria possui irmãos no mesmo acolhimento institucional. Constatou-se baixa escolaridade dos pais e a sua inserção em atividades laborais informais, ou ainda, desemprego. Embora não se possa precisar a atual configuração familiar, o fato de que em mais de $70 \%$ dos casos os pais dos participantes não viviam mais juntos sugere mobilidade e/ou instabilidade quanto às

Tabela 3. Médias e desvios-padrão da Escala de Satisfação Multidimensional de Vida (ESMV) e das variáveis do Mapa dos Cinco Campos por sexo e faixa etária.

\begin{tabular}{|c|c|c|c|c|c|c|c|c|c|c|}
\hline & \multicolumn{2}{|c|}{ Meninos } & \multicolumn{2}{|c|}{ Meninas } & \multicolumn{2}{|c|}{ Crianças } & \multicolumn{2}{|c|}{ Adolescentes } & \multicolumn{2}{|c|}{ Total } \\
\hline & M & $\mathrm{DP}$ & M & $\mathrm{DP}$ & M & $\mathrm{DP}$ & M & $\mathrm{DP}$ & M & DP \\
\hline ESMV & 3,9 & 0,5 & 3,8 & 0,5 & 3,9 & 0,5 & 3,8 & 0,5 & 3,8 & 0,5 \\
\hline Contatos Satisfatórios & 26,7 & 12 & 31,4 & 16,5 & 28,3 & 14,5 & 29,6 & 14,7 & 29,1 & 14,5 \\
\hline Contatos Insatisfatórios & 2,2 & 2,5 & 2,9 & 3,1 & 2,2 & 2,9 & 2,7 & 2,7 & 2,5 & 2,8 \\
\hline Conflito & 6,5 & 5,2 & 8,5 & 6,8 & 5,7 & 4,5 & $9,29 *$ & 6,9 & 7,5 & 6,1 \\
\hline Rompimento & 3,4 & 3,4 & $5,5^{*}$ & 5,6 & 3,6 & 3,5 & $5,18 * *$ & 5,5 & 4,4 & 4,7 \\
\hline Campo Família & 6,9 & 3,6 & 8,4 & 5,7 & 7,6 & 4,7 & 7,7 & 4,9 & 7,7 & 4,8 \\
\hline Campo Abrigo & 8,3 & 5 & 10,2 & 6,6 & 9,0 & 5,9 & 9,5 & 5,9 & 9,3 & 5,9 \\
\hline Campo Escola & 5,6 & 3,7 & 6,8 & 4,6 & 6,0 & 3,5 & 6,3 & 4,8 & 6,2 & 4,2 \\
\hline Campo Amigos/Parentes & 5,5 & 4,9 & 5,1 & 4,4 & 5,2 & 4,9 & 5,4 & 4,5 & 5,3 & 4,7 \\
\hline Campo Contatos Formais & 2,7 & 2,3 & 3,8 & 4,1 & 2,9 & 2,3 & 3,6 & 4,1 & 3,3 & 3,4 \\
\hline Total & 29,2 & 12,5 & $34,3^{* *}$ & 17,2 & 31,1 & 14,9 & 32,3 & 15,5 & 31,7 & 15,2 \\
\hline
\end{tabular}

Nota: ${ }^{*} p<0,01 ; * * p<0,05$ 
Tabela 4. Médias e desvios-padrão do fator de proximidade por sexo e faixa etária.

\begin{tabular}{lcccccccccc}
\hline \multirow{2}{*}{ Campo } & \multicolumn{2}{c}{ Meninos } & \multicolumn{2}{c}{ Meninas } & \multicolumn{2}{c}{ Crianças } & \multicolumn{2}{c}{ Adolescentes } & \multicolumn{2}{c}{ Total } \\
\cline { 2 - 11 } & M & DP & M & DP & M & DP & M & DP & M & DP \\
\hline Família & 6,7 & 1,6 & 6,3 & 1,8 & 6,6 & 1,9 & 6,4 & 1,6 & 6,5 & 1,7 \\
Abrigo & 5,8 & 2,0 & 5,5 & 1,9 & 5,8 & 2,1 & 5,5 & 1,8 & 5,7 & 2,0 \\
Escola & 5,7 & 2,3 & 5,1 & 2,2 & 5,6 & 2,3 & 5,3 & 2,3 & 5,4 & 2,2 \\
Amigo & 5,4 & 2,6 & 5,5 & 2,6 & 5,2 & 2,9 & 5,7 & 2,3 & 5,5 & 2,6 \\
Contatos Formais & 5,6 & 2,9 & 5,5 & 2,7 & 5,9 & 2,7 & 5,2 & 2,9 & 5,6 & 3,0 \\
\hline Total & 6,0 & 1,4 & 6,0 & 1,4 & 6,0 & 1,5 & 5,8 & 1,3 & 6,5 & 1,7 \\
\hline
\end{tabular}

configurações familiares. Esse perfil indica a precariedade econômica e social dessas famílias, que apresentam indicadores de risco presentes na realidade brasileira, como alto índice de casamento, divórcio e recasamento, baixa escolaridade e atividades informais de trabalho (Instituto Brasileiro de Geografia e Estatística - IBGE, 2008).

Ainda quanto ao microssistema familiar, pode-se constatar que a maioria dos participantes mantinha contato semanal com a família, demonstrando que essas crianças e adolescentes possuíam referência familiar. A pesquisa realizada por Silva (2004), em acolhimentos institucionais de todo o Brasil, também apontou que 58\% dos abrigados tinham contato com seus familiares. Esse resultado confirma que apesar do vínculo existente entre eles, essas famílias não têm conseguido exercer seu papel protetivo junto a suas crianças e adolescentes, que acabam sendo encaminhadas para instituições de proteção.

Considerando as características da pessoa do PPCT, foi verificada experimentação de drogas lícitas e ilícitas entre os participantes. O álcool já foi experimentado pela maioria dos participantes, seguido do cigarro e das drogas ilícitas. A análise apontou um início precoce, com experimentação a partir de cinco anos de idade. A experimentação de álcool e drogas ilícitas foi mais frequente entre os meninos e a experimentação de cigarro, entre as meninas, além de ambos serem mais frequentes entre os adolescentes. Estudo desenvolvido em 2004 com crianças e adolescentes abrigados e em conflito com a lei, de idade semelhante à dessa amostra, na cidade de Porto Alegre (Ferigolo \& cols., 2004), indicou média de experimentação de álcool, cigarro e drogas ilícitas superior à média encontrada neste estudo (médias de idade de 11,7; 11,8 e 12,4 anos, respectivamente). Esses resultados mostram a precoce exposição desses jovens com relação às drogas, e confirmam a situação de risco na qual esses jovens encontravam-se, mesmo antes de serem institucionalizados, tendo em vista que a droga é um importante fator de risco para o desenvolvimento.

Os participantes do estudo apresentaram baixo desempenho escolar, visualizado pela pouca escolaridade $(46,4 \%$ estavam cursando $2^{\mathrm{a}}$ e $3^{\mathrm{a}}$ séries do Ensino Fundamental), alto índice de repetência $(72,7 \%)$ e baixos escores no TDE. As meninas apresentaram escores maiores que os meninos tanto no escore total do TDE quanto dos subtestes, demonstrando melhor desempenho escolar. Considerando a média total do instrumento, observou-se que esses jovens apresentaram um desempenho escolar inferior ao encontrado em outro estudo, que utilizou o mesmo instrumento com crianças e adolescentes de mesma faixa etária e de nível socioeconômico desfavorecido que viviam com suas famílias também no sul do Brasil (Machado, 2006). Ferreira e Marturano (2002) destacam que fatores como problemas nas relações interpessoais, falhas parentais quanto à supervisão, monitoramento e suporte, indícios de menor investimento dos pais no desenvolvimento das crianças, práticas punitivas e modelos adultos agressivos estão presentes entre as crianças com baixo desempenho escolar. Dell'Aglio e Hutz (2004) também encontraram um menor desempenho escolar entre as crianças institucionalizadas quando comparadas às que viviam com a família, demonstrando a importância da família para o desempenho escolar na infância.

A análise da EMSV apontou médias próximas de 4 (cujo valor máximo é 5), indicando boa satisfação de vida. Esse resultado pode sugerir que as condições de vida atuais desses jovens estão lhes proporcionando bem-estar e satisfação de vida. O funcionamento dos acolhimentos institucionais tem se modificado após o advento do ECA (Brasil, 1990), especialmente no que diz respeito à individualização do atendimento, à redução do espaço e ao número de abrigados e à preocupação com a convivência familiar e comunitária. Outro aspecto que poderia influenciar na satisfação de vida está relacionado ao fato de que muitos jovens abrigados têm acesso, no acolhimento institucional, a condições de vida que não teriam se estivessem com suas famílias, como educação, lazer, vestuário, entre outros, além de estarem afastados das situações de violência e abandono que levaram à institucionalização. Dessa forma, esses fatores - melhorias nas instituições, vivência de violência no contexto de origem e condições socioeconômicas desfavorecidas nas famílias - podem contribuir para a percepção de satisfação de vida no abrigo. No entanto, cabe destacar que essa percepção positiva diminui com a idade, tendo em vista que foi observada uma correlação negativa entre satisfação de vida e idade. Isso pode indicar que os adolescentes apresentam maior capacidade de avaliar a situação que estão vivendo, talvez com maior capacidade de reflexão e crítica, do que os mais jovens. Esse dado é importante para as estratégias de acompanhamento a essa população, reiterando a importância da provisoriedade da medida de proteção. 
A análise da rede de apoio forneceu informações sobre a dimensão contexto do modelo bioecológico. Cada campo do instrumento pode ser considerado um microssistema, contexto no qual o indivíduo mantém relações face a face. Observou-se que o Campo Abrigo sobressaiu-se em termos de estrutura, à medida que apresentou maior quantidade de contatos dos que outros campos, e função, visto que apresentou fator de proximidade de grande força. Esse resultado, associado ao alto escore de satisfação de vida, indica que o abrigo pode estar exercendo um papel importante na rede de apoio para essas crianças e adolescentes, promovendo desenvolvimento. Outros estudos (Dalbem \& Dell'Aglio, 2008; Siqueira \& cols., 2006; Yunes \& cols., 2004) também apontam que, no acolhimento institucional, os jovens encontram apoio junto aos pares e aos profissionais, podendo a instituição operar como fator de proteção, proporcionando confiança, segurança e a possibilidade de compartilhar sentimentos.

Por outro lado, o fator de proximidade no Campo Família também foi de grande força, sugerindo relações próximas e com qualidade nesse microssistema, apesar do afastamento. A literatura tem destacado que muitas crianças e adolescentes institucionalizados apresentam uma percepção otimista e positiva da família, atribuindo-lhe qualidades, minimizando os pontos negativos e os fatores que levaram à institucionalização (Siqueira \& cols., 2009; Yunes \& Szymanski, 2003).

Relações entre o tempo de institucionalização e variáveis investigadas podem ser destacadas: maior tempo de institucionalização esteve correlacionado à menor média de contatos no Campo Abrigo; menor média do fator de proximidade no Campo Abrigo e menor média de contatos no Mapa. Ressalta-se que esses resultados devem ser considerados com cautela, pois apesar de as correlações terem sido significativas, elas foram baixas. Entretanto, eles sugerem que o tempo de institucionalização pode influenciar a rede de apoio, especialmente quanto à percepção de proximidade e qualidade das relações. No cotidiano de muitas instituições, constata-se a existência de grande rotatividade tanto dos funcionários/ técnicos, a partir das constantes admissões e licenças laborais, quanto das outras crianças e adolescentes abrigados, em função de seus desligamentos ou mesmo de fugas. Esses fatores podem provocar uma "couraça" nesses jovens, fazendo com que, ao longo do tempo, eles se envolvam e invistam menos nas relações interpessoais no interior da instituição, com o objetivo de proteger-se de mais sofrimento no momento da separação. Dessa forma, por mais que evidências indiquem aspectos positivos relacionados ao abrigamento, sugerindo que esse contexto esteja funcionando como um fator de proteção, os dados deste estudo sugerem efeitos do tempo nesse processo e, por isso, a necessidade de o abrigamento ser excepcional e provisório, conforme preconiza o ECA. Além disso, o estudo de Dell'Aglio e Hutz (2004) já havia destacado que o tempo de institucionalização influencia negativamente no desempenho escolar, corroborando os efeitos prejudiciais de um longo período de abrigamento.

As diferenças entre sexo e faixa etária na rede de apoio social e afetivo estão em consonância com a literatura da área. Neste estudo, as meninas apresentaram mais contatos na rede, conflitos e rompimentos do que os meninos, embora com efeitos de baixa magnitude. A média significativamente maior de conflitos entre as meninas abrigadas também foi apontada por Siqueira e cols. (2006), podendo indicar que as meninas podem apresentar maior vulnerabilidade a perdas. Para Rudolph e Hammen (1999), as meninas investem mais do que os meninos em seus relacionamentos, como fonte de apoio emocional e de identidade pessoal, levando-as a sentir o estresse interpessoal como uma ameaça ao seu bem-estar. Assim, as meninas experimentam níveis de estresse interpessoal mais altos do que os meninos, especialmente em conflitos com os pais, pares e companheiros.

A maior média de conflitos e rompimentos entre os adolescentes, em comparação às crianças, pode ser explicada pela fase de vida que estão vivenciando. A adolescência é considerada uma fase do ciclo vital que envolve inúmeras mudanças, tanto físicas, emocionais, cognitivas quanto sociais, que requerem ajustes e adaptações internas adicionais. Essas mudanças relacionadas à puberdade podem fazer com que os adolescentes vivenciem um período de maior vulnerabilidade aos conflitos e problemas cotidianos (Adams, Overholser \& Spirito, 1994; Steinberg, 1999). Muitos dos eventos estressantes vividos pelos adolescentes estão relacionados à aquisição e transição de papéis como, por exemplo, adaptação a novos ambientes, a diferentes demandas acadêmicas e a mudanças nas relações com pares do mesmo sexo ou do sexo oposto (Gröer, Thomas \& Schoffner, 1992).

\section{Considerações Finais}

A análise dos dados deste estudo forneceu importantes informações sobre variáveis individuais e familiares de crianças e adolescentes institucionalizados em acolhimentos institucionais da Região Metropolitana de Porto Alegre, considerando as dimensões pessoa e contexto do modelo bioecológico (Bronfenbrenner, 2004; Bronfenbrenner \& Morris, 1998). Foi possível constatar que grande parte dos familiares dos participantes possuía baixa escolaridade, desenvolvia atividade informal de trabalho e os pais não viviam mais juntos. A maioria dos participantes mantinha contato com seus familiares, entre eles, avós, irmãos mais velhos, além de pais e mães. Indícios de vulnerabilidade e risco foram observados, como o uso de álcool e drogas com início precoce, alto índice de repetência, baixa escolaridade e baixos escores de desempenho escolar. Esses resultados mostram a necessidade de desenvolvimento de programas de reforço escolar a fim de estimular o desenvolvimento cognitivo dessa população, para que o déficit escolar não seja mais um elemento excludente para as crianças e adolescentes abrigados, além de ações de prevenção em relação ao uso de drogas.

Os resultados deste estudo sugerem que o tempo de institucionalização pode trazer prejuízos, especialmente quanto à percepção de proximidade na rede de apoio. Apesar de o abrigamento ser uma medida de proteção excepcional e temporária, é possível observar que muitas crianças e adolescentes permanecem por muitos anos institucionalizados, embora mantenham contato com a família. Torna-se importante a criação de mecanismos sociais que promovam a provisoriedade dessa medida de proteção, a partir de ações conjuntas entre as instituições e as famílias, para que um planejamento seja traçado com vistas ao retorno familiar, 
de forma que os efeitos de um período prolongado numa instituição sejam diminuídos. Para melhor compreender os efeitos do tempo de institucionalização para o desenvolvimento de crianças e adolescentes, faz-se necessário desenvolver pesquisas que focalizem esse aspecto, com delineamento longitudinal, com amostras maiores, que permitam um acompanhamento dessas crianças e adolescentes, assim como uma avaliação comparativa de crianças que permanecem por períodos mais curtos ou por longos períodos no acolhimento institucional.

Destaca-se que as características apresentadas neste estudo se referem à Região Metropolitana de Porto Alegre, e embora a amostra tenha abarcado $82 \%$ da população de jovens abrigados com idades entre 7 e 16 anos nessa região, no período do estudo, seus resultados não podem ser generalizados para outros contextos e para outras instituições. No entanto, o conhecimento do perfil dessa população possibilita que as entidades responsáveis pelos jovens afastados de suas famílias planejem ações e atividades de intervenção, de forma que tenham condições de propiciar o seu desenvolvimento.

\section{Referências}

Adams, D. M., Overholser, J. C., \& Spirito, A. (1994). Stressful life events associated with adolescent suicide attempts. Canadian Journal of Psychiatry, 39, 43-48.

Arpini, D. (2003). Violência e exclusão: adolescência em grupos populares. São Paulo: EDUSC.

Brasil. (1990). Estatuto da criança e do adolescente. Lei $n^{o}$ 8069, de 13 de julho de 1990. Brasília: Diário Oficial da União.

Brasil (2009). Lei $n^{\circ} 12.010$, de 3 de agosto de 2009. Retirado em 09/03/2010,de http://www.planalto.gov.br/ccivil_03/_Ato20072010/2009/Lei/L12010.htm.

Brito, R., \& Koller, S. H. (1999). Desenvolvimento humano e redes de apoio social e afetivo. Em A. Carvalho (Ed.), O mundo social da criança: natureza e cultura em ação (pp. 115-129). São Paulo: Casa do Psicólogo.

Bronfenbrenner, U. (1996). A ecologia do desenvolvimento humano. Porto Alegre: Artes Médicas. (Trabalho original publicado em 1979)

Bronfenbrenner, U. (2004). Making human beings human: Biecological perspectives on human development. London: Sage.

Bronfenbrenner, U., \& Morris, P. (1998). The ecology of developmental processes. Em W. Damon (Ed.), Handbook of child psychology (pp. 993-1027). New York: John Wiley \& Sons.

Cohen, J. (1998). Statistical power analysis for the behavioral sciences. Hillsdate: Lawrence Earlbaum.

Dalbem, J. X., \& Dell'Aglio, D. D. (2008). Apego em adolescentes institucionalizadas: processos de resiliência na formação de novos vínculos afetivos. Psico (PUCRS), 39, 33-40.

De Antoni, C., \& Koller, S. H. (2000). A visão de famílias entre as adolescentes que sofreram violência intrafamiliar. Estudos de Psicologia, 5, 347-381.

Dell'Aglio, D. (2000). O processo de coping, institucionalização e eventos de vida em crianças e adolescentes. Tese de Doutorado, Universidade Federal do Rio Grande do Sul, Porto Alegre.

Dell'Aglio, D., \& Hutz, C. (2004). Depressão e desempenho escolar em crianças e adolescentes institucionalizados. Psicologia: Reflexão e Crítica, 17, 341-350.
Ferigolo, M., Barbosa, F., Arbo E., Malysz, A., Stein, A., \& Barros, H. (2004). Prevalência do consumo de drogas na FEBEM, Porto Alegre. Revista Brasileira de Psiquiatria, 26, 10-16.

Ferreira, M., \& Marturano, E. (2002). Ambiente familiar e os problemas de comportamento apresentados por crianças com baixo desempenho escolar. Psicologia: Reflexão e Crítica, 15, 35-44.

Garmezy, N., Masten, A. S., \& Tellegen, A. (1984). The study of stress and competence in children: A building block for developmental psychopathology. Child Development, 55, 97-111.

Giacomoni, C. (2002). Bem-estar subjetivo infantil: conceito de felicidade e construção de instrumentos para avaliação. Tese de Doutorado, Universidade Federal do Rio Grande do Sul, Porto Alegre.

Giacomoni, C., \& Hutz, C. S. (2008). Escala multidimensional de satisfação de vida para crianças: estudos de construção e validação. Estudos de Psicologia, 25, 23-35.

Gröer, M. W., Thomas, S. P., \& Schoffner, D. (1992). Adolescence stress and coping: A longitudinal study. Research in Nursing and Health, 15, 209-217.

Guará, I. M. F. F. (2006). Abrigo: comunidade de acolhida e socioeducação. Em M. V. Baptista (Ed.), Abrigo: comunidade de acolhida e socioeducação (pp. 63-75). São Paulo: Instituto Camargo Corrêa.

Hoppe, M. (1998). Redes de apoio social e afetivo de crianças em situação de risco. Dissertação de Mestrado, Universidade Federal do Rio Grande do Sul, Porto Alegre.

Instituto Brasileiro de Geografia e Estatística - IBGE (2008). Censo Nacional - Instituto Brasileiro de Geografia e Estatística. Retirado em 27/01/2010, de http:/www.ibge.gov.br/home/ presidencia/noticias/17122003registrocivilhtml.shtm.

Lucas, R., Diener, E., \& Suh, E. (1996). Discriminate validity of well-being measures. Journal of Personality and Social Psychology, 71, 616-628.

Machado, P. X. (2006). Impacto e processo de um projeto de educação pelo esporte no desenvolvimento infantil. Dissertação de Mestrado, Universidade Federal do Rio Grande do Sul, Porto Alegre.

Martins, E., \& Szymanski, H. (2004). Brincando de casinha: significado de família para crianças institucionalizadas. Estudos de Psicologia, 9, 177-187.

Masten, A., \& Garmezy, N. (1985). Risk, vulnerability and protective in developmental psychopathology. Em B. Lahey \& A. Kazdin (Ed.), Advances in clinical child psychology (pp.1-52), New York: Plenum.

Oliveira, R. C. (2006). A história começa a ser revelada: panorama atual do abrigamento no Brasil. Em M. V. Baptista (Ed.), Abrigo: comunidade de acolhida e socioeducação (pp. 39-51). São Paulo: Instituto Camargo Corrêa.

Pasian, S., \& Jacquemin, A. (1999). O autorretrato em crianças institucionalizadas. Paidéia, 9, 50-60.

Rizzini, I., \& Rizzini, I. (2004). A institucionalização de crianças no Brasil. Rio de Janeiro: PUC-Rio.

Rudolph, K. D., \& Hammen, C. (1999). Age and gender as determinants of stress exposure, generation, and reactions in youngsters: A transactional perspective. Child Development, 70, 660-677.

Ryff, C. D., \& Keyes, C. L. (1995). The structure of psychological well-being revisited. Journal of Personality and Social Psychology, 69, 719-727. 
Samuelsson, M., Thernlund, G., \& Ringström, J. (1996). Using the five field map to describe the social network of children: A methodological study. International Journal Behavioral Development, 19, 327-345.

Silva, E. R. (2004). O direito à convivência familiar e comunitária: os abrigos para crianças e adolescentes no Brasil. Brasília: IPEA/CONANDA.

Siqueira, A. C., Betts, M. K., \& Dell'Aglio, D. D. (2006). Rede de apoio social e afetivo de adolescentes institucionalizados. Interamerican Journal of Psychology, 40, 149-158.

Siqueira, A. C., \& Dell'Aglio, D. D. (2006). O impacto da institucionalização na infância e na adolescência: uma revisão de literatura. Psicologia \& Sociedade, 18, 71-80.

Siqueira, A. C., Tubino, C. L., Schwarz, C., \& Dell'Aglio, D. D. (2009). Família e institucionalização: percepção das figuras parentais na rede de apoio de jovens institucionalizados. Arquivos Brasileiros de Psicologia, 61, 176-190.

Sluzki, C. (1997). A rede social na prática sistêmica: alternativas terapêuticas. São Paulo: Casa do Psicólogo.
Stein, L. (1994). Teste de desempenho escolar (TDE): manual para aplicação e interpretação. São Paulo: Casa do Psicólogo.

Steinberg, L. (1999). Adolescence. Boston: McGraw-Hill.

Yunes, M. A., Miranda, A., \& Cuello, S. (2004). Um olhar ecológico para os riscos e as oportunidades de desenvolvimento de crianças e adolescentes institucionalizados. Em S. H. Koller (Ed.), Ecologia do desenvolvimento humano: pesquisa e intervenções no Brasil (pp. 197-218). São Paulo: Casa do Psicólogo.

Yunes, M., A. M., \& Szymanski, H. (2003). Crenças, sentimentos e percepções acerca da noção de resiliência em profissionais da Saúde e Educação que atuam com famílias pobres. Psicologia da Educação, 17, 119-137.

Recebido em 14.07.08

Primeira decisão editorial em 15.01.10

Versão final em 19.07.10

Aceito em 19.07.10 